\title{
A Molecular Disk Survey of Low-Mass Stars in the TW Hya Association
}

\author{
David R. Rodriguez ${ }^{1}$, Gerrit van der Plas ${ }^{1,8}$, Joel H. Kastner ${ }^{2}$, Adam \\ C. Schneider ${ }^{3}$, Jacqueline K. Faherty ${ }^{4,5}$, Diego Mardones ${ }^{1}$, Subhanjoy \\ Mohanty $^{6}$, and David Principe ${ }^{7,8}$ \\ ${ }^{1}$ Departamento de Astronomía, Universidad de Chile, Casilla 36-D, Santiago, Chile \\ ${ }^{2}$ Center for Imaging Science, School of Physics \& Astronomy, and Laboratory for \\ Multiwavelength Astrophysics, Rochester Institute of Technology, Rochester, NY 14623, USA \\ ${ }^{3}$ Department of Physics and Astronomy, University of Toledo, Toledo, OH 43606, USA \\ ${ }^{4}$ Department of Terrestrial Magnetism, Carnegie Institution of Washington, Washington, DC \\ 20015, USA \\ ${ }^{5}$ Hubble Fellow \\ ${ }^{6}$ Imperial College London, London SW7 2AZ, UK \\ ${ }^{7}$ Núcleo de Astronomía, Facultad de Ingeniería, Universidad Diego Portales, Santiago, Chile \\ ${ }^{8}$ Millennium Nucleus Protoplanetary Disks, Chile
}

\begin{abstract}
We have carried out an ALMA Cycle 2 survey of 15 confirmed or candidate low-mass $\left(<0.2 M_{\odot}\right)$ members of the TW Hya Association (TWA) with the goal of detecting line emission from $\mathrm{CO}$ molecular gas and continuum emission from cold dust. Our targets have spectral types of M4-L0 and hence represent the extreme low end of the TWA's mass function. The survey has yielded a detection of ${ }^{12} \mathrm{CO}(2-1)$ emission around TWA 34 . This newly discovered $\sim 10$ Myr-old molecular gas disk lies just $\sim 50 \mathrm{pc}$ from Earth.
\end{abstract}

Keywords. open clusters and associations: individual (TWA) - protoplanetary disks - stars: evolution — stars: pre-main sequence

\section{Introduction}

Beginning with the identification of the TW Hya Association (TWA) several stellar associations with ages $\sim 8-200$ Myr have been identified in close proximity to the Earth (Zuckerman \& Song 2004, Torres et al. 2008). These young moving groups serve as excellent laboratories to explore the evolution of stellar and planetary properties. Of particular interest is the growing number of $\mathrm{M}$ dwarfs identified as members of these groups and how the lifetimes of protoplanetary disks orbiting such low-mass stars compare with those of higher mass members.

To this end, we have carried out an Atacama Large Millimeter Array (ALMA) survey of 15 recently identified very low-mass members or candidate members of the TWA. Our sample is drawn from Looper et al. (2007, 2010a,b), Looper (2011), Shkolnik et al. (2011), Rodriguez et al. (2011), Schneider et al. (2012), and Gagné et al. (2004). Several are known to host dusty, circumstellar disks as inferred from WISE and Herschel infrared excesses, but none had yet been observed with ALMA.

Our ALMA Cycle 2 program (2013.1.00457.S) consisted of observations of continuum dust emission at $230 \mathrm{GHz}$ and observations of the ${ }^{12} \mathrm{CO}(2-1)$ and ${ }^{13} \mathrm{CO}(2-1)$ emission lines with a velocity resolution of $0.6 \mathrm{~km} / \mathrm{s}$. The requested angular resolution was $1.6^{\prime \prime}$ as we did not aim to resolve any emission. We reached a sensitivity of $0.05 \mathrm{mJy} / \mathrm{beam}$ in the continuum and $5 \mathrm{mJy} /$ beam per $0.6 \mathrm{~km} / \mathrm{s}$ channel in ${ }^{12} \mathrm{CO}$ and ${ }^{13} \mathrm{CO}$. Calibration and cleaning was performed by the ALMA staff with CASA version 4.2.2. 


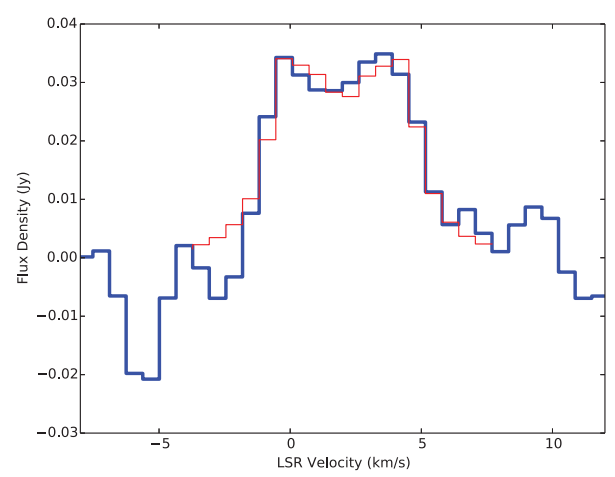

Figure $1 .{ }^{12} \mathrm{CO}(2-1)$ emission line profile of the gas around TWA 34, the only target in our sample with detected $\mathrm{CO}$ gas. The red, thin line is a best-fit Keplerian profile.

\section{Results}

We report unambigious $1.3 \mathrm{~mm}$ continuum detections of four objects (TWA 30B, 32, 33, 34). We only detected ${ }^{12} \mathrm{CO}$ emission toward TWA 34 . In Fig. 1, we show the integrated line profile of the CO emission, Hanning smoothed with a kernel size of 3 channels. To characterize this emission, we fit a parametrized Keplerian model as described in Kastner et al. (2008). We obtain an integrated intensity of $0.34 \pm 0.03 \mathrm{Jy} \mathrm{km} / \mathrm{s}$. Combined with our 3- $\sigma$ upper limit on ${ }^{13} \mathrm{CO}$ emission of $\sim 0.03 \mathrm{Jy} \mathrm{km} / \mathrm{s}$, we estimate a gas mass of $\sim 0.2 M_{E}$, following the methods of Kastner et al. (2008) and assuming $T_{e x}=40 \mathrm{~K}$, a ${ }^{12} \mathrm{C}:{ }^{13} \mathrm{C}$ ratio of 89 , and a $\mathrm{CO}: \mathrm{H}_{2}$ ratio of $10^{-4}$.

With our best-fit Keplerian profile, we can estimate a systemic velocity for the CO emission. In the barycentric frame of reference, this corresponds to $13.3 \pm 0.1 \mathrm{~km} / \mathrm{s}$. This is consistent with membership in TWA.

\section{Conclusions}

We have carried out an ALMA survey of 15 low-mass TWA members and candidates to search for molecular gas in the form of ${ }^{12} \mathrm{CO}$ and ${ }^{13} \mathrm{CO}$ as well as continuum dust emission. We detect continuum emission from four objects. Only one system, TWA 34, shows signatures of molecular gas in its disk in the form of ${ }^{12} \mathrm{CO}(2-1)$ emission. TWA 34 is a new low-mass star hosting a molecular gas disk at just $50 \mathrm{pc}$ from Earth. The systemic velocity we measure for the circumstellar CO around TWA 34 is consistent with membership in the TWA.

\section{References}

Gagné, J., Faherty, J. K., Cruz, K., et al. 2014, ApJ Letterse, 785, LL14

Kastner, J. H., Zuckerman, B., \& Forveille, T. 2008, A\&AA, 486, 239

Looper, D. L., Burgasser, A. J., Kirkpatrick, J. D., \& Swift, B. J. 2007, ApJ Letters, 669, L97

Looper, D. L., Mohanty, S., Bochanski, J. J., et al. 2010, ApJ, 714, 45

Looper, D. L., Bochanski, J. J., Burgasser, A. J., et al. 2010b, AJ, 140, 1486

Looper, D. L. 2011, Ph.D. Thesis, University of Hawai'i

Rodriguez, D. R., Bessell, M. S., Zuckerman, B., \& Kastner, J. H. 2011, ApJ, 727, 62

Roeser, S., Demleitner, M., \& Schilbach, E. 2010, AJ, 139, 2440 754, 39

Schneider, A., Song, I., Melis, C., Zuckerman, B., \& Bessell, M. 2012, ApJ, 757, 163

Shkolnik, E. L., Liu, M. C., Reid, I. N., Dupuy, T., \& Weinberger, A. J. 2011, ApJ, 727, 6

Torres, C. A. O., et al. 2008, Handbook of Star Forming Regions, Volume II, 757

Zuckerman, B. \& Song, I. 2004, ARA\&A, 42, 685 